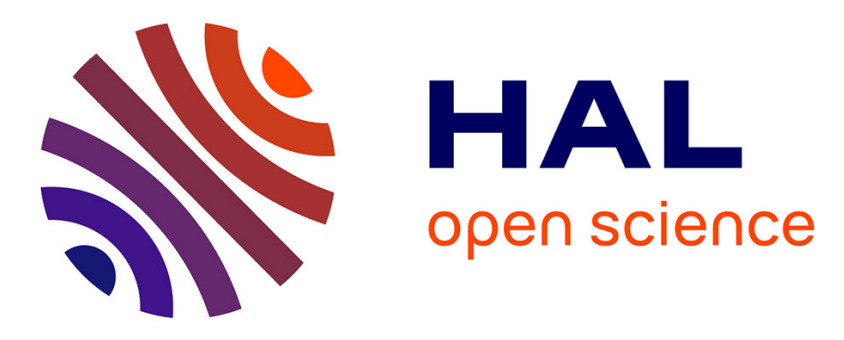

\title{
A Decision Support System to design modified atmosphere packaging for fresh produce based on a bipolar flexible querying approach
}

Valérie Guillard, Patrice Buche, Sébastien Destercke, Nouredine Tamani, Madalina Croitoru, Luc Menut, Carole Guillaume, Nathalie Gontard

\section{To cite this version:}

Valérie Guillard, Patrice Buche, Sébastien Destercke, Nouredine Tamani, Madalina Croitoru, et al.. A Decision Support System to design modified atmosphere packaging for fresh produce based on a bipolar flexible querying approach. Computers and Electronics in Agriculture, 2015, 111, pp.131-139. 10.1016/j.compag.2014.12.010 . hal-01104835

\section{HAL Id: hal-01104835 \\ https://hal.science/hal-01104835}

Submitted on 19 Jan 2015

HAL is a multi-disciplinary open access archive for the deposit and dissemination of scientific research documents, whether they are published or not. The documents may come from teaching and research institutions in France or abroad, or from public or private research centers.
L'archive ouverte pluridisciplinaire HAL, est destinée au dépôt et à la diffusion de documents scientifiques de niveau recherche, publiés ou non, émanant des établissements d'enseignement et de recherche français ou étrangers, des laboratoires publics ou privés. 
- A Decision Support System to design

. modified atmosphere packaging for fresh

${ }_{5} \quad$ Valérie Guillard ${ }^{1}$, Patrice Buche*2, Sébastien Destercke ${ }^{3}$,

6 Nouredine Tamani ${ }^{4}$, Madalina Croitoru ${ }^{4}$, Luc Menut ${ }^{2}$, Carole

7 produce based on a bipolar flexible querying approach
${ }^{1}$ UMR IATE INRA UM2, 2 Place Eugène Bataillon Montpellier, France. \{guillard, guillaume, gontard\}@um2.fr

${ }^{2}$ UMR IATE INRA, 2 place Pierre Viala Montpellier, France. \{patrice.buche, luc.menut\}@supagro.inra.fr ${ }^{*}$ Corresponding author. Tel. +33 499612283 ; fax. +33 499613076.

${ }^{3}$ UMR7253 Heudiasyc, CNRS, UTC Centre de recherches de Royallieu, F-60205 Compiègne, France. sebastien.destercke@hts.utc.fr ${ }^{4}$ INRIA Graphik LIRMM, 161 rue Ada Montpellier, France tamani@lirmm.fr, ntamani@gmail.com, croitoru@lirmm.fr 


\begin{abstract}
To design new packaging for fresh food, stakeholders of the food chain express their needs and requirements, according to some goals and objectives. These requirements can be gathered into two groups: (i) fresh food related characteristics and (ii) packaging intrinsic characteristics. Modified Atmosphere Packaging (MAP) is an efficient way to delay senescence and spoilage and thus to extend the very short shelf life of respiring products such as fresh fruits and vegetables. Consequently, packaging $\mathrm{O}_{2} / \mathrm{CO}_{2}$ permeabilities must fit the requirements of fresh fruits and vegetable as predicted by virtual MAP simulating tools. Beyond gas permeabilities, the choice of a packaging material for fresh produce includes numerous other factors such as the cost, availability, potential contaminants of raw materials, process ability, waste management constraints, etc. For instance, the user may have the following multi-criteria query for his/her product asking for a packaging with optimal gas permeabilities that guarantee product quality and optionally a transparent packaging material made from renewable resources with a cost for raw material less than $3 € / \mathrm{kg}$. To help stakeholders taking a rational decision based on the expressed needs, a new multi-criteria Decision Support System (DSS) for designing biodegradable packaging for fresh produce has been built. In this paper we present the functional specification, the software architecture and the implementation of the developed tool. This tool includes (i) a MAP simulation module combining mass transfer models and respi-
\end{abstract}


ration of the food, (ii) a multi-criteria flexible querying module which handles imprecise, uncertain and missing data stored in the database. We detail its operational functioning through a real life case study to determine the most satisfactory materials for apricots packaging.

Keywords. MAP modeling, multi-criteria querying, decision support system, knowledge engineering, respiring product.

\section{Introduction}

Despite targeted campaigns and programs for promoting their health benefits, consumption of fresh fruits and vegetable is still limited by their short shelf life, which is not easily compatible with current modes of distribution and purchase (once a week) in medium- to high-income countries.

Beyond respect of the chill chain and initial food quality, Modified Atmosphere Packaging (MAP) was proved to be an efficient way to delay senescence and spoilage, without using controversial preservative compounds, and so to extend shelf life of fresh produce [Floros and Matsos, 2005, Guillaume et al., 2008, Zagory and Kader, 1988]. MAP relies on the establishment of an optimal / recommended atmosphere for the produce and can be achieved by matching the gas $\left(\mathrm{O}_{2}\right.$ and $\left.\mathrm{CO}_{2}\right)$ permeation rate of the film with the respiration rate of the produce. If a wealth of information has been published on MAP (more than 400 occurrences for "modified atmosphere packaging and fruit and vegetable" in ISI Web of Knowledge), there is a lack of systematic treatment of the data 
using knowledge management system in order to provide a full (complete) Decision Support System (DSS).

By providing suitable information, such numerical tools could help designers and users to select film properties that best fit particular purposes and targets. This approach is especially important when developing packaging made from biodegradable materials, which becomes a new trend, as their limited barrier properties, possibly optimized using smart and/or composite multilayer material [Guillaume et al., 2010], can turn out to be an asset to extend shelf life of respiring foods [Cagnon et al., 2012, Guilbert et al., 2011, Guillaume et al., 2008]. To facilitate MAP design, mathematical models, socalled virtual MAP, have been developed by researchers working in this field to design passive [Mahajan et al., 2007, Souza-Gallagher and Mahajan, 2013] or active MAP [Cagnon et al., 2012, Charles et al., 2003, Charles et al., 2005] for fresh and fresh-cut fruits and vegetable. Online applications are today available for free (www.tailorpack.com) or charged access (www.packinmap.com). Such numerical tools simplify the package design steps by predicting the gas permeability values that permit to reach recommended atmosphere for the target product and therefore to extend shelf life while maintaining quality and safety of the packed food.

Up to now, all the aforementioned tools only considered the gas permeabilities of the packaging material as a basis for packaging design. The design of food packaging is not only driven by maximizing shelf life of the food, and numerous other requirements may interplay for final decision, re- 
lated to processing, marketing, commercial, or distribution concerns (as cost, process ability of constituents, industrial feasibility, environmental impact, safety and stability of the packaging material all over the food life cycle, waste management, etc.). Then, aside the constraint of food shelf life and quality, correlated to gas permeation rates of the material, it also relies on user's preferences, naturally expressed as wishes (e.g. transparent material would be preferred) or constraints (e.g. cost of raw material must be less than $3 € / \mathrm{kg})$. Some constraints and/or wishes are also related to the fact that consumers may reject the use of some additives or of nano-technology in the packaging material because of the unknown consequences on their health, or more simply they may prefer transparent rather than opaque packaging. To help stakeholders of the food chain in the choice of a packaging material that would suit all the requirements of a given fresh fruit or vegetable, development of decision aid tools is foreseen. 


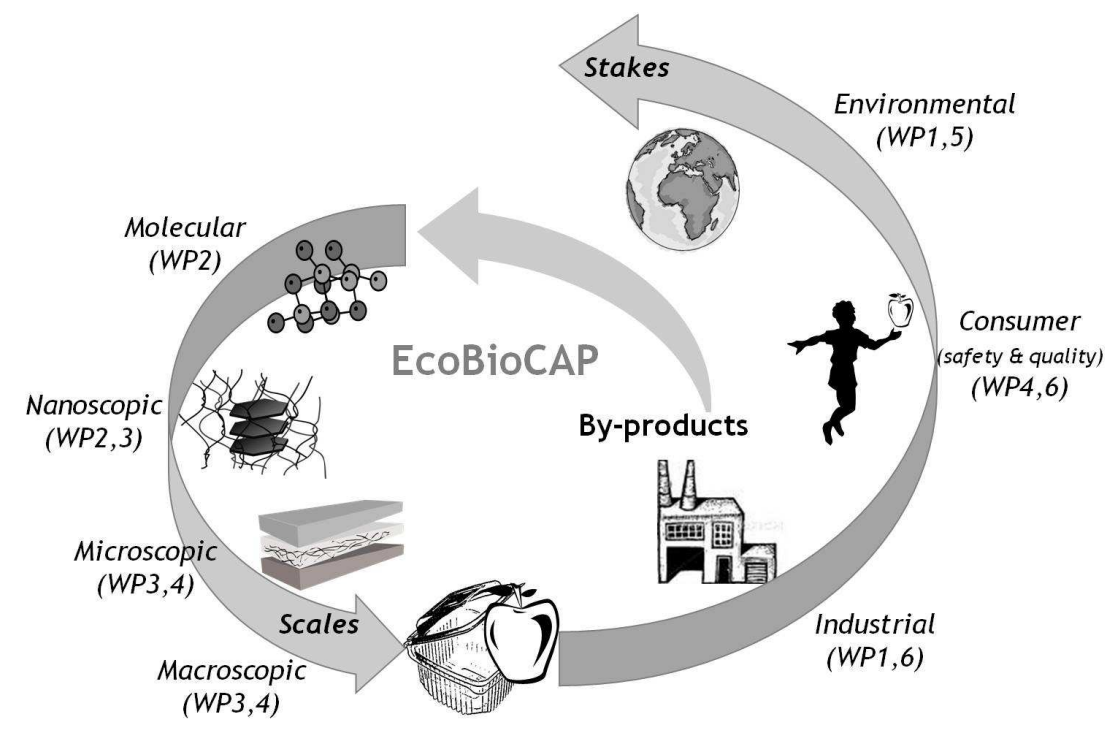

Figure 1: The EcoBioCap project.

The European project EcoBioCap (www.ecobiocap.eu) aims at designing the next generation of packaging material using advanced composite structures based on constituents derived from the food industry (cf figure 1). In the framework of this project, we aim at developing a Decision Support System (DSS) to help parties involved in the packaging design to make rational decisions based on knowledge expressed by the experts of the domain. The Decision Support System developed in this context aims at solving the dilemma of multi-criteria demands in the design of packaging for fresh produce. This DSS relies on the development of a querying system (i) able to store and maintain data in dedicated databases (which could be incomplete or imprecise) about packaging material characteristics (e.g. gas permeabilities, cost, transparency, mechanical properties, etc.) and fresh food parameters 
112

(e.g. respiration parameters, optimal storage conditions), (ii) allowing stakeholders to express their needs and requirements as queries addressed to the system databases, and (iii) retrieving the packaging materials ranked from the most to the least satisfactory according to the expressed requirements.

The original contribution of this paper is to detail the design and the implementation of the proposed Flexible and Bipolar Multi-criteria Querying system, part of the DSS for the EcoBioCap project:

- Functional description of the system based on the approach described in [Destercke et al., 2011],

- Databases development and filling with food and packaging characteristics,

- Open source implementation with $\mathrm{Java}^{1}, \mathrm{R}$ for numerical processing ${ }^{2}$, and MySQL ${ }^{3}$ relational database management system.

Functional specifications of the bipolar flexible querying system are introduced in Section 2. Its corresponding software architecture is detailed in Section 3. Implementation aspects and some tests are displayed in Section 4. Section 5 concludes the paper.

\footnotetext{
${ }^{1}$ www.java.com

2 http://www.r-project.org

${ }^{3}$ www.mysql.com
} 


\section{Functional specifications of the system}

We detail here the main functions that the bipolar flexible querying system has to provide to the users. Prospective users of the EcoBioCap DSS have been identified in the Stakeholder Advisory Board of the EcoBioCap FP7 project.

Functional requirements implemented in the EcoBioCap DSS are the following:

1. Collecting and managing data available about the packaging material characteristics,

2. Collecting and managing data available about the respiration parameters of fresh produce,

3. Managing users' preferences expressed over packaging material targeted characteristics as constraints or wishes,

4. Dealing with missing data, since in real cases some required packaging characteristics could be unknown, so stakeholders of the food chain may face the problem of missing data,

5. Managing imprecise data, since characteristics associated with packaging materials (eg. $\mathrm{O}_{2}$ permeability values) and food products (eg. maximal respiration rates) may be imprecise, 
6. Retrieving the ranked list of all relevant packaging with their main characteristics,

7. Guaranteeing the retrieval of packagings which are the closest to the requirements (called guaranteed solutions) in case of empty set of solutions,

\section{Architecture of the flexible querying system}

Figure 2 details the components of the developed flexible querying system implementing the required functionalities. Namely, (i) two databases (for fresh foods and packaging materials), (ii) the virtual MAP simulation and (iii) the multi-criteria flexible querying system. 


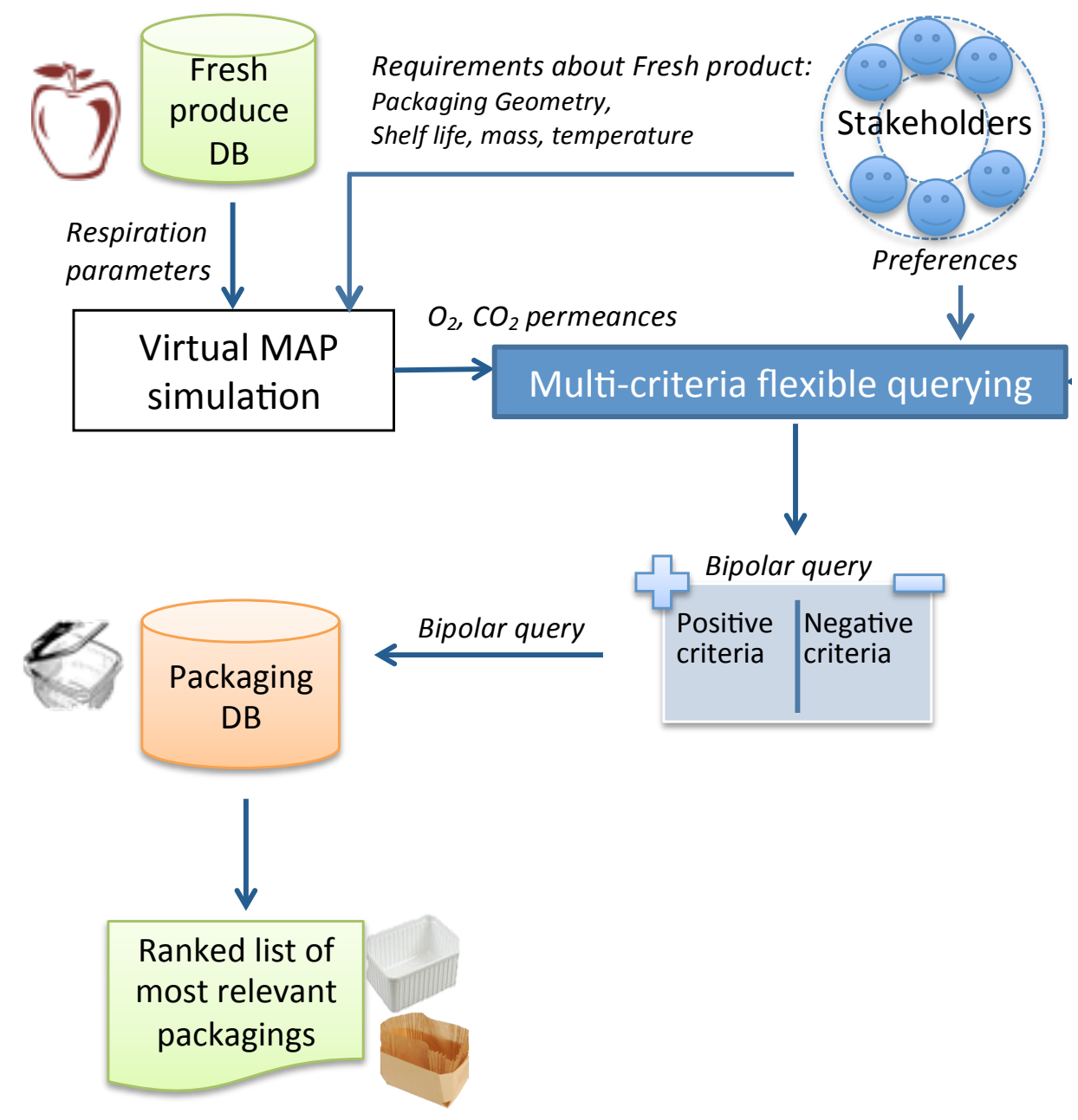

Figure 2: The flexible querying architecture.

\subsection{Databases}

The flexible querying system defines and implements two databases: (i) fresh food database containing the respiration parameters of the fresh produce used in the virtual MAP simulation (maximal respiration rate, Mickaëlis-Menten 
constant, energy of activation, optimal gas concentration required for the fresh produce optimal conservation, respiratory quotient), and (ii) packaging materials database storing all the data related to the packaging material: $\mathrm{O}_{2}$ and $\mathrm{CO}_{2}$ permeabilities, temperature at which the permeability measurement was done, film thickness, mechanical properties, indication about the cost, the renew-ability or not of the raw material used for producing the packaging film, the biodegradability, the transparency, etc. Both databases contain informations collected from the literature in the field and they are maintained within MySQL (www.mysql.com) RDBMS (Relational DataBase Management System).

Whenever available, each datum is stored with its confidence interval to allow uncertainty propagation during simulations.

\subsection{The virtual MAP module}

The virtual MAP simulation module computes the optimal permeance which guaranties the best shelf life for the packed food, by combining data from the fresh food database and parameters given by the user. Inputs extracted from the fresh food database are:

- parameters of the Mickaëlis-Menten type equations: maximal respiration rate $R \max$, respiratory quotient $R Q$, Mickaëlis-Menten constant $K m$, etc.

- optimal $\mathrm{O}_{2}$ and $\mathrm{CO}_{2}$ content targeted in the packaging headspace when 
running simulation.

Parameters given by the user include the targeted shelf life, the food mass to pack, the storage temperature, and the geometric dimensions of the packaging (surface and volume). By using mass transfer mathematical equations (based on Fick's law) coupled with Mickaëlis-Menten equation for respiration, the virtual MAP returns the optimal $\mathrm{O}_{2}$ and $\mathrm{CO}_{2}$ permeances and permeabilities for the preservation of the food.

The virtual MAP module has been implemented using the R software for numerical computing (http://www.r-project.org).

\subsection{The multi-criteria flexible querying module}

To build such a querying system, methodologies based on Flexible MultiCriteria Querying process were used. The needs and requirements are modeled as user preferences, approach widely studied in the field of flexible querying of databases and information retrieval systems. Flexible querying systems allow users expressing preferences in their queries. These queries are run on regular relational databases and deliver a set of discriminated answers, which are ranked from the most to the least preferred. Preferences are usually expressed as ordinary and binary sets. But they may lead to empty set of answers and enlarged intervals of preferences relaxing the constraints could be more expressive than a single value. In this context, fuzzy sets theory [Zadeh, 1965] provides a general model for the interpretation of queries in- 
volving preferences. The querying system [Destercke et al., 2011] can also cope with lack of data or imprecise data in the database corresponding to the characteristics related to the packagings like the optimal permeance, the dimension of the packaging, its shape, etc.

Besides, expressed user preferences have different levels of importance or priority. The shelf life and sanitary criteria ensuring a good preservation of the packed product are intuitively more important than the color or the transparency of the packaging. Therefore, some preferences are modeled as constraints that the satisfaction is mandatory, and some others are wishes that satisfaction is optional. Any packaging material which does not satisfy the constraints is definitely discarded and the more packaging satisfies the wishes the more preferred it is. It is natural then to make use in this context of a bipolar approach for the querying process since it permits to handle compound preferences made of mandatory conditions and optional conditions. Bipolarity refers to the human reasoning which combines pro and con information to take decisions, to make choices or judgments. It has been widely studied during the last years in the field of preference modeling for flexible querying. Several approaches have been introduced for the expression and the evaluation of fuzzy bipolar conditions [Bordogna and Pasi, 1994, Dubois and Prade, 2002b, Dubois and Prade, 2002a, de Tré et al., 2009], [Zadrozny and Kacprzyk, 2007, Zadrozny and Kacprzyk, 2009, Liétard et al., 2011, Tamani et al., 2013]. In this paper, we consider fuzzy bipolar conditions as a particular case of fuzzy conditions, and we rely on the interpretation intro- 
duced by Dubois and Prade [Dubois and Prade, 2002b, Dubois and Prade, 2002a, Dubois and Prade, 2008], in which a bipolar condition is made of constraints that are a mandatory condition (which refer to the negative pole or con information) and wishes that are optional conditions (which refer to the positive pole or pro information), and globally expressing "constraints, and if possible wishes".

Moreover, the main difference of our flexible querying approach in comparison with the aforementioned ones is that in our case we take into consideration the fact that some values associated with packaging materials attributes can be imprecise or uncertain, since they were obtained from experimental data returned from repetitions corresponding to an interval instead of a single value. Therefore, as for query preferences, values in the database are also considered as fuzzy sets, but with a semantic adapted to imprecise data instead of preferences, as defined in [Dubois and Prade, 1997, Haemmerlé et al., 2007, Buche et al., 2011].

Predicted optimal gas permeances, computed by the virtual MAP module become two input parameters corresponding to one criterion (considered by default as constraints since related to food quality, but could be also switched to wishes in the $\mathrm{GUI}^{4}$ ) in the multi-criteria flexible querying module.

The multi-criteria flexible querying module combines these inputs to form a bipolar query addressed to the packaging database. The returned list of packagings is ranked from the most to the least relevant one with regard to

\footnotetext{
${ }^{4}$ Graphical User Interface
} 
the expressed preferences. The user can finally specify whether the ranking has to take into account unknown values for the mandatory criteria. If the ranking must consider unknown values for the mandatory criteria, then each delivered packaging is annotated with the percentage of known values over which the ranking was carried out. In the opposite case, if the ranking must only consider known values for the mandatory criteria and if no packaging in the database has the required characteristics, (leading to empty set answer), then the system activates the guaranteed result function which computes the most similar packagings or the closest packagings to the ideal one.

As previously stated, the flexible querying system relies on a bipolar approach handling fuzzy conditions to model user preferences expressing pro and con informations.

\section{Fuzzy sets theory:}

The fuzzy sets theory ${ }^{5}$ is introduced by Zadeh [Zadeh, 1965] to express the gradual membership of an element to a set. A fuzzy set generalizes a crisp set in which membership grades are in $\{0,1\}$. If a fuzzy set is a discrete set then it is denoted $F=\left\{\left(x_{1}, \mu_{F}\left(x_{1}\right)\right), \ldots,\left(x_{n}, \mu_{F}\left(x_{n}\right)\right)\right\}$, otherwise it is characterized by its membership function, generally a trapezoidal function. For instance, Figure 3 illustrates the membership (trapezoidal) function $\mu_{\mathrm{PeO}_{2}}$

\footnotetext{
${ }^{5}$ Formally, a fuzzy set $F$ is defined on a referential $U$ by a membership function $\mu_{F}$ : $U \mapsto[0,1]$, such that $\mu_{F}(x)$ denotes the membership grade of $x$ in $F$. In particular, $\mu_{F}(x)=1$ denotes the full membership of $x$ in $F, \mu_{F}(x)=0$ expresses the absolute nonmembership and when $0<\mu_{F}(x)<1$, it reflects a partial membership (the closer to 1 $\mu_{F}(x)$, the more $x$ belongs to $\left.F\right)$.
} 
corresponding to an $\mathrm{O}_{2}$ permeance preference.

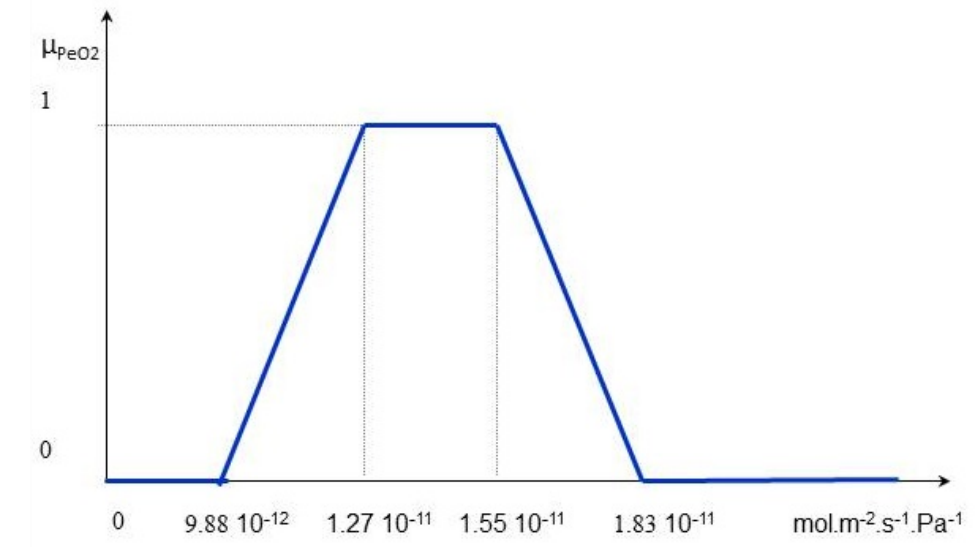

Figure 3: Example of a fuzzy set corresponding to $\mathrm{O}_{2}$ permeance preference.

269

The union $\cup$ and the intersection $\cap$ operators are defined with a couple of (t-norm, t-conorm) such as $(\min , \max )$. Let $F, G$ be two fuzzy sets, $\mu_{F \cup G}(x)=\max \left(\mu_{F}(x), \mu_{G}(x)\right), \mu_{F \cap G}(x)=\min \left(\mu_{F}(x), \mu_{G}(x)\right)$, and the complement of $F$, denoted $F^{c}$, is defined by $\mu_{F^{c}}(x)=1-\mu_{F}(x)$.

The logical counterparts of $\cap, \cup$ and the complement are respectively $\wedge$ (AND operator), $\vee($ OR operator) and $\neg$ (negation operator).

\section{The flexible bipolar querying method:}

The flexible bipolar querying method implemented in the DSS corresponds to a 'pros and cons' approach. Criteria chosen by the user (wishes and/or constraints) are multiple and also sorted by importance.

More formally, a bipolar query is pair of a sorted combination of contraints and wishes of the form $Q=(C, W)$, where $C=\left\{C_{(1)}, \ldots, C_{(n)}\right\}$ and $W=$ 


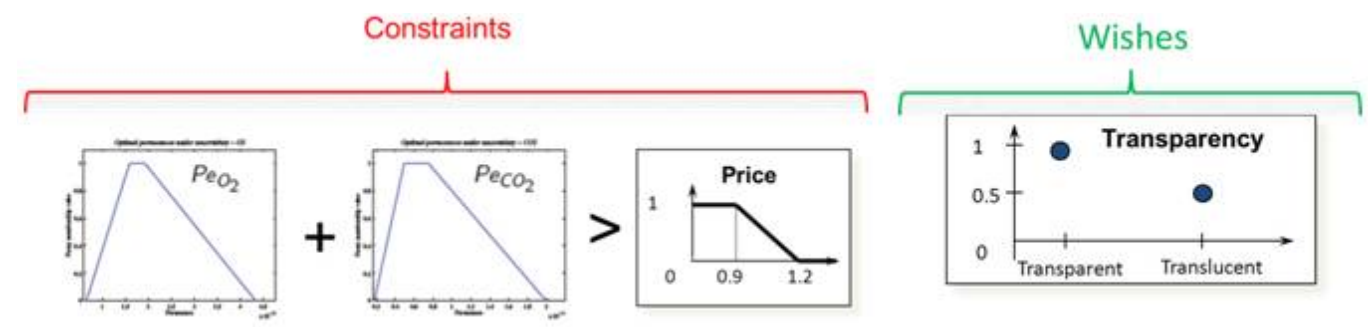

$\left\{W_{(1)}, \ldots, W_{(m)}\right\}$; each $C_{i, i=1, \ldots, n}$ (resp. $\left.W_{j, j=1, \ldots, m}\right)$ is a subset of contraints (resp. wishes) of the same importance or priority expressed on the attributes of packaging material such that:

- $\forall i, i^{\prime} \in\{1, \ldots, n\}$, if $i<i^{\prime}$ then contraints in $C_{(i)}$ take priority over $C_{\left(i^{\prime}\right)}$

- $\forall j, j^{\prime} \in\{1, \ldots, m\}$ if $j<j^{\prime}$, wishes in $W_{(j)}$ take priority over $W_{\left(j^{\prime}\right)}$

An example of such a query with two constraints and one wish is represented as a sorted combination of fuzzy sets displayed in Figure 4. It corresponds to the following query:

$$
\begin{aligned}
& Q\left(C=\left\{\left\{\text { Pre } f_{\text {O2Permeance }}, \text { Pre } f_{\text {CO2Permeance }}\right\}\right.\right.\left.,\left\{\text { Pre } f_{\text {Price }}\right\}\right\}, \\
&\left.W=\left\{\left\{\text { Pre } f_{\text {Transparency }}\right\}\right\}\right) .
\end{aligned}
$$

Figure 4: Example of a bipolar query.

Handling imprecise and uncertain values. Considering the food packaging application, enlarged intervals for the values could be taken into account (e.g. uncertainties due to biological variability [Destercke et al., 2011] or tolerance threshold expressed by the user). In the flexible querying system, as for query preferences, values in the database are also considered as fuzzy 
sets, but with a semantic adapted to imprecise data instead of preferences, as defined in [Dubois and Prade, 1997].

Thanks to the homogeneous representation of preferences and uncertain values, the comparison between a fuzzy set having a semantic of preference with a fuzzy set having a semantic of imprecision can be defined using two classical measures of possibility theory: a possibility degree of matching (denoted $\Pi$ ) and a necessity degree of matching (denoted $N$ ) [Dubois and Prade, 1988] defined as follows:

- the possibility degree of matching between two fuzzy sets $v$ and $v^{\prime}$ defined on a referential domain Dom, denoted $\Pi\left(v, v^{\prime}\right)$, is $\Pi\left(v, v^{\prime}\right)=$ $\operatorname{Sup}_{x \in \operatorname{Dom}}\left(\min \left(\mu_{v}(x), \mu_{v}^{\prime}(x)\right)\right)$,

- the necessity degree of matching between $v$ and $v^{\prime}$ denoted $N\left(v, v^{\prime}\right)$, is $N\left(v, v^{\prime}\right)=$ $\operatorname{In} f_{x \in \operatorname{Dom}}\left(\max \left(\mu_{v}(x), 1-\mu_{v}^{\prime}(x)\right)\right)$ (see Figure 5 for a graphical representation). 


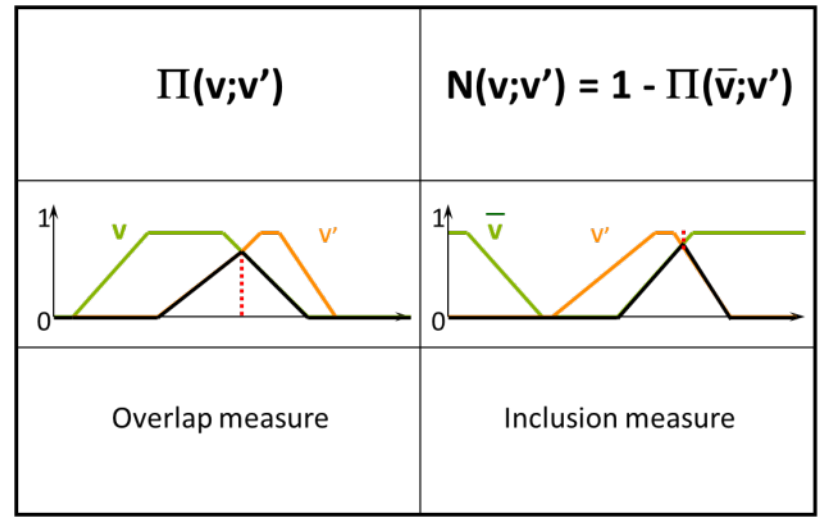

Figure 5: Example of overlapping and inclusion measures.

Intuitively, the possibility degree of matching is an optimistic comparison, as it corresponds to the maximum degree that satisfies simultaneously the membership function of the two fuzzy sets (representing preferences and uncertainty, respectively). The necessity degree may be considered as a pessimistic comparison, as it corresponds to the inverse of the degree of inclusion of the fuzzy set associated with the uncertainty interpretation (i.e., our knowledge stored in the database) with the one associated with the preferences.

When two criteria $C_{1}$ and $C_{2}$ of the query are of equal priority, the respective couples of comparison degrees $\left[N_{C_{1}}, \Pi_{C_{1}}\right]$ and $\left[N_{C_{2}}, \Pi_{C_{2}}\right]$, for a given material stored in the database, are aggregated to provide a unique couple of comparison degrees $\left[N_{C_{1} \cap C_{2}}, \Pi_{C_{1} \cap C_{2}}\right]$ where $N_{C_{1} \cap C_{2}}=\min \left(N_{C_{1}}, N_{C_{2}}\right)$ and $\Pi_{C_{1} \cap C_{2}}=\min \left(\Pi_{C_{1}}, \Pi_{C_{2}}\right)$. At the end, the algorithm provides a ranking of equivalent classes: the first one contains the best materials that are of similar 
quality and the last one contains those materials that are the less satisfactory while still fulfilling the constraints. For further details about the ranking process, the reader is referred to [Destercke et al., 2011].

Handling missing data. It's worth noticing that those comparison degrees may be evaluated even if, for a given material stored in the database, some data are missing. For any fuzzy sets of preferences, comparison degrees with packaging materials for which data are missing in the database will be equal to $[N=0, \Pi=1]$.

That means that, when a material stored in the database is associated with a missing datum, the DSS will consider that this material could potentially match the food requirement related to this criteria $(\Pi=1)$ and, simultaneously, that the degree of necessity that this material match the food requirement is equal to $0(N=0)$.

Handling empty set as answer. In the case where every packaging material has a possibility degree of matching of 0 for at least one constraint (no material is candidate), the DSS warns the user and searches the materials which are the closest, according to some distance, to satisfy the constraints.

That is, for each material $M$ in the database, the system computes a distance $d(M, C)$ between the material and the constraints $C$, and orders the materials according to this distance. This distance can be seen as the minimal modification the constraints $C$ would have to undergo so that material $M$ satisfies it (see [Destercke et al., 2011] for more details). Finally, the user is 
informed that the specified constraints are probably too restrictive but still receives a list of materials that almost satisfy his/her needs.

\subsection{Validation of the flexible querying system}

The validation of the results delivered by the DSS has been done twofold. Firstly, it relied on the validation of the virtual MAP model which has been done in the past on endives and tomatoes [Charles et al., 2003, Charles et al., 2005, Cagnon et al., 2012, Cagnon et al., 2013]. Secondly, it has been done using a classical use-cases testing procedure of the functionalities associated with the flexible bipolar querying system presented in section 3.3. This validation has been done by involving stakeholders of the food chain participating to the EcoBioCap project. Some collective testing sessions have been organized in 2012 and 2013 in order to validate by the potential users the functionalities of the DSS. Following these testing sessions, the users have requested the implementation of two possible behaviors of the database flexible querying concerning missing data handling. The first request corresponds to the one described in section 3.3 in which, to inform the user, a percentage of known values corresponding to the querying criteria has been added to each answer. The second request corresponds to the desire of having a more restrictive querying mode forbidding the ranking of packagings with unknown values. Consequently, now, both modes of querying are available in the DSS. 
367

\section{Implementation}

The flexible querying system was implemented as a web application accessible on http://pfl.grignon.inra.fr/EcoBioCapQuerying/. A short demonstration video is available for download on

http://umr-iate.cirad.fr/axes-de-recherche/ingenierie-des-connaissances/themesde-recherche/ecobiocap-dss.

The interface of the application is made of 3 parts:

- the upper part, shown on Figure 6, is dedicated to the permeance simulation and allows the setting of the fresh food and packaging parameters. It is connected to the fresh food database to retrieve the characteristics associated with the selected fresh food. Figure 6 displays in the upper part the optimal permeance properties for the apricot Bergeron, computed by the DSS, for a shelf life of 7 days in ambient temperature $\left(20^{\circ} \mathrm{C}\right)$, mass food of $0.5 \mathrm{~kg}$, volume of $1 \mathrm{l}$ and surface of $756 \mathrm{~cm}^{2}$.

- the middle part allows the user expressing his/her preferences. In this version, the user can only specify his/her preferences about the O2/CO2 permeances, the storage temperature, the biodegradability and the transparency of the packaging material. The text of the multicriteria querying shown on Figure 6 would be : "I want a packaging material that suits to my product, apricot (eg. its O2 and CO2 permeances match the apricot requirement) for the range of temperature between 14 and $26^{\circ} \mathrm{C}$ ". It must be noticed that the optimal permeances 

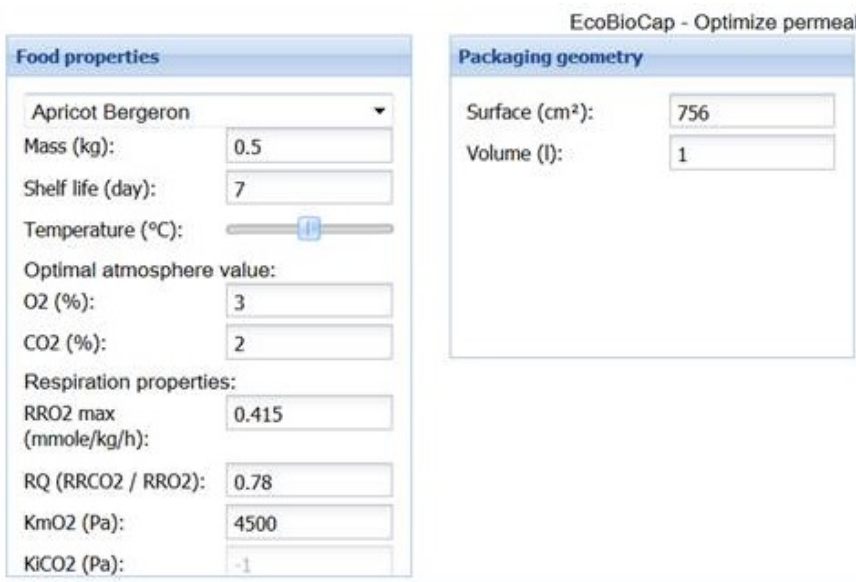

Preferences associated with criteria

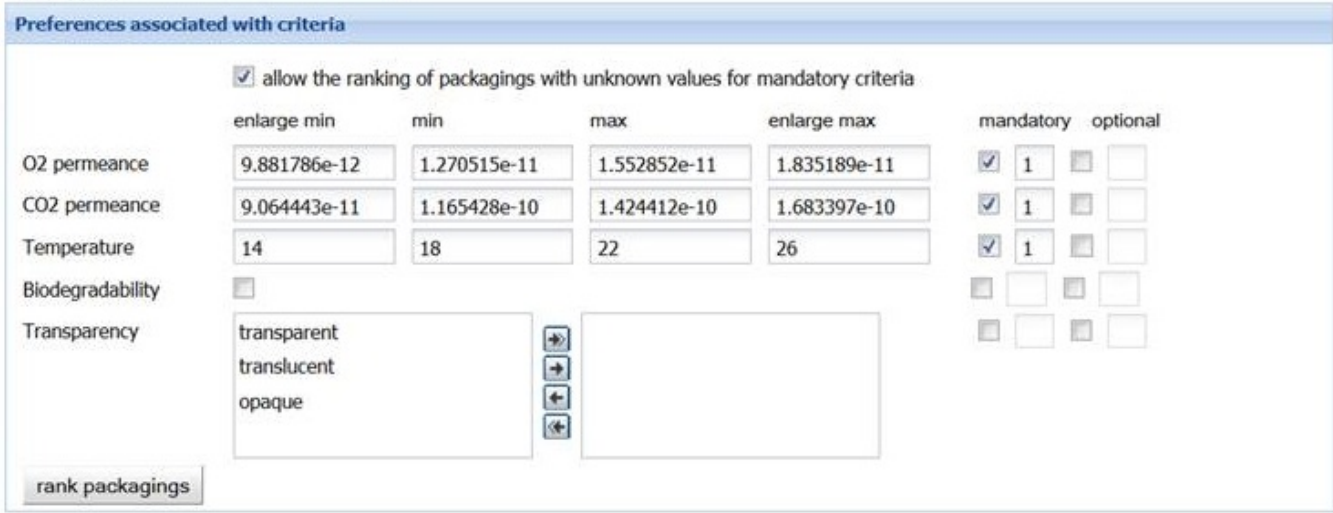

Figure 6: Permeance values obtained in the case of Apricot.

- The lower part is dedicated to the result of the query, as shown in computed by the DSS are automatically replicated in the middle part with a predefined deviation for the min-max and enlarge min-max intervals. Those values correspond to the fuzzy preferences associated with permeances as presented in Figure 3. They may be modified by the user before launching the querying of the packaging database.

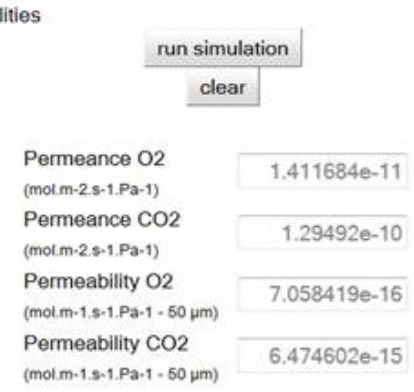

run simulation
Figure 7 in the case of Apricot. Please notice that in this example, 


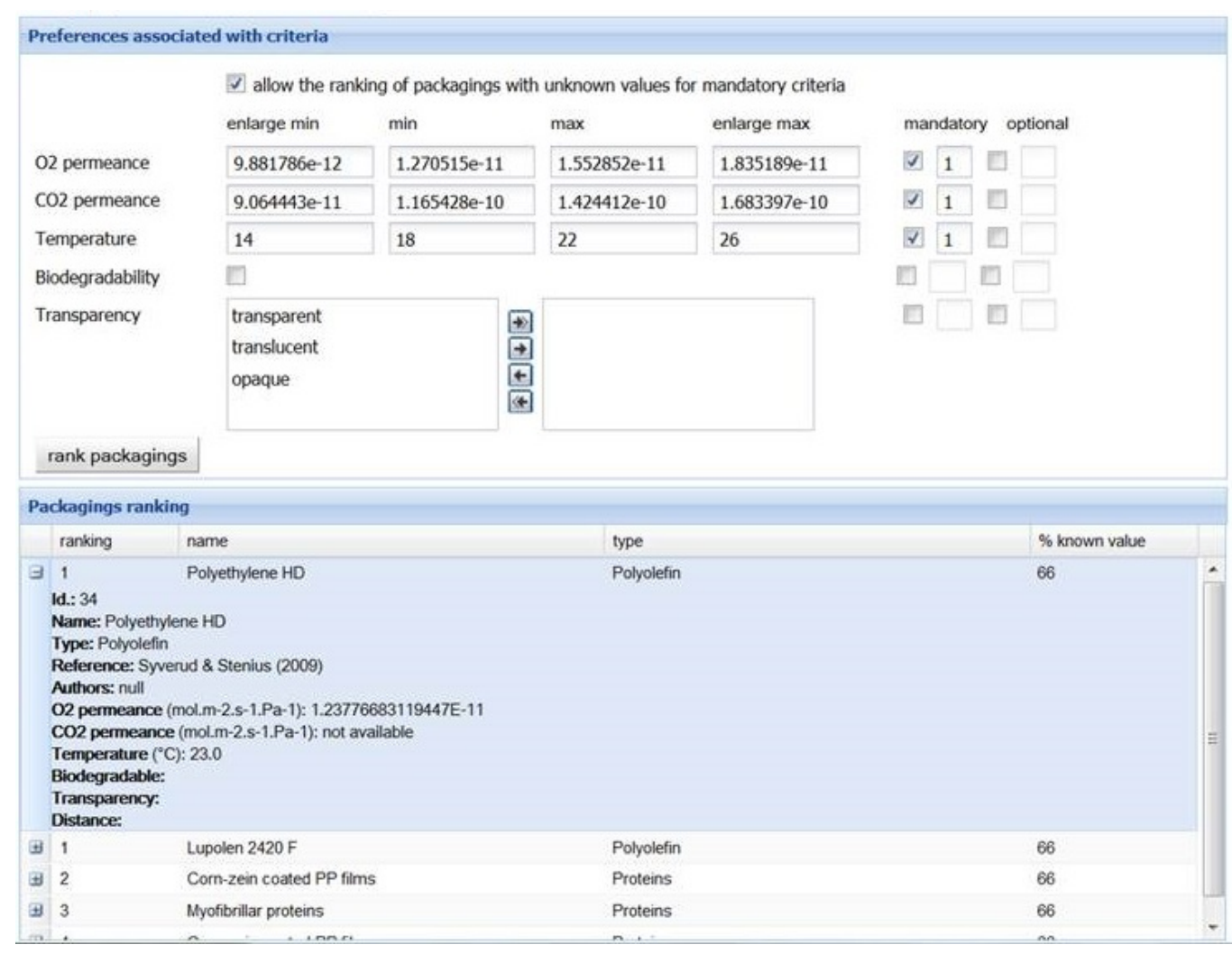

Figure 7: Ranked list of suitable packaging in the case of Apricot (only constraints are considered). Details are displayed for the first packaging.

only constraints are considered and the process allows the ranking of the packaging with unknown values for mandatory criteria (the highest percentage of known values in the ranking is $66 \%$ ).

Further details (commercial packaging or not, available reference of the scientific publication from where the information has been extracted, etc.) about delivered packaging materials can also be displayed as illustrated in Figure 7 for the same case of Apricot. 
The DSS considers not only mandatory criteria such as packaging permeabilities but also wishes of the user. For instance the user may have the following query: "I want a packaging material that permits to preserve the shelf life of my product (Apricot) in the range of temperature $14-26^{\circ} \mathrm{C}$ and if possible biodegradable". This query corresponds to three constraints: $\mathrm{O} 2$ and $\mathrm{CO} 2$ permeabilities and temperature and one wish. Figure 8 illustrates this query. We notice that the wish permits to break tie between packagings having the same ranking with regard to constraints. In the present case study (Apricot), the two materials with number 1 in Figure 7 where polyolefins, oil-based materials, which are not biodegradable. Therefore the segregation between them shown in Figure 8 is not made on their biodegradability characteristics but on the fact that in one case, this criterion is filled in the database (case of lupolen, become rank 2 in the new classification) and in the case of polyethylene (rank 1), this value is not filled and thus considered as potentially reachable (cf section 3.3, paragraph Handling missing data). The user is informed about lacking data thanks to the column $\%$ known values. By example, for the case of polyethylene, it is $50 \%$ as only two values are known (see Figure 8): the O2 permeance and the temperature. 


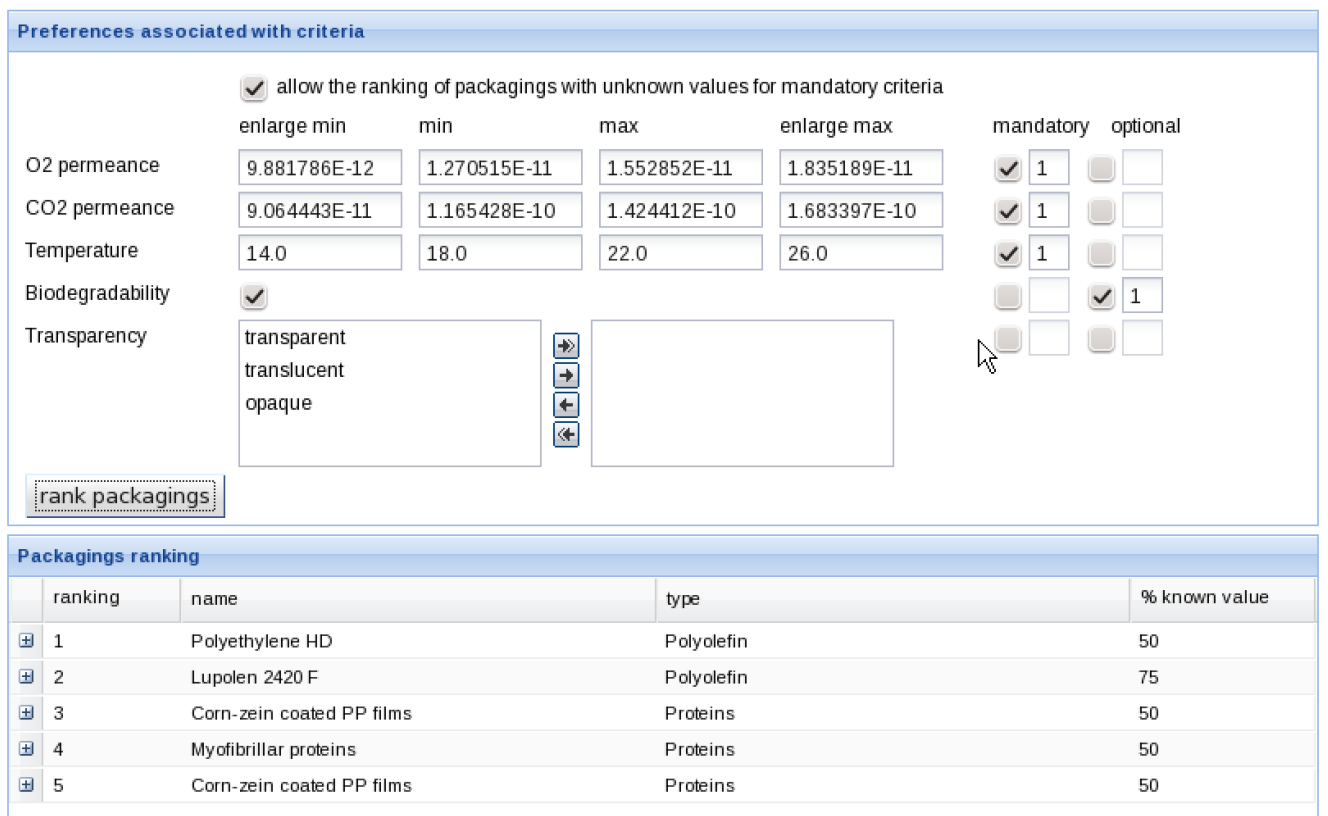

Figure 8: Ranked list of suitable packagings in the case of Apricot (a wish is also considered).

Finally, Figure 9 illustrates the guaranteed result function of the DSS which delivers the closest packaging materials according to a distance calculated between the query constraints and the actual value of those materials. The guaranteed result function is launched when there are not actually any packaging having all the criteria values fulfilled in the packaging database and satisfying the query constraints. In the query corresponding to the case study of Figure 9, the criteria biodegradability is not a wish but a constraint. As no packaging material of the database fully satisfies this query, the guaranteed result function 
is computed. A warning is sent to the user through a pop-up window (see Figure 9) in which is indicated, for each mandatory criterion, the percentage of packagings which do not fulfill the constraint or have an unknown value. The ranking of packaging materials proposed to the user is made with the packagings which are the closest to the targeted ones (constraints in the query). In the present case study, as biodegradability became mandatory, the first ranked material is a biodegradable one "wheat gluten" even if its $\mathrm{O} 2 / \mathrm{CO} 2$ permeabilities are further from the target than those of polyehylene, the material selected in the first query (Figure 7). This last query well illustrates how the DSS could always guarantee some results.

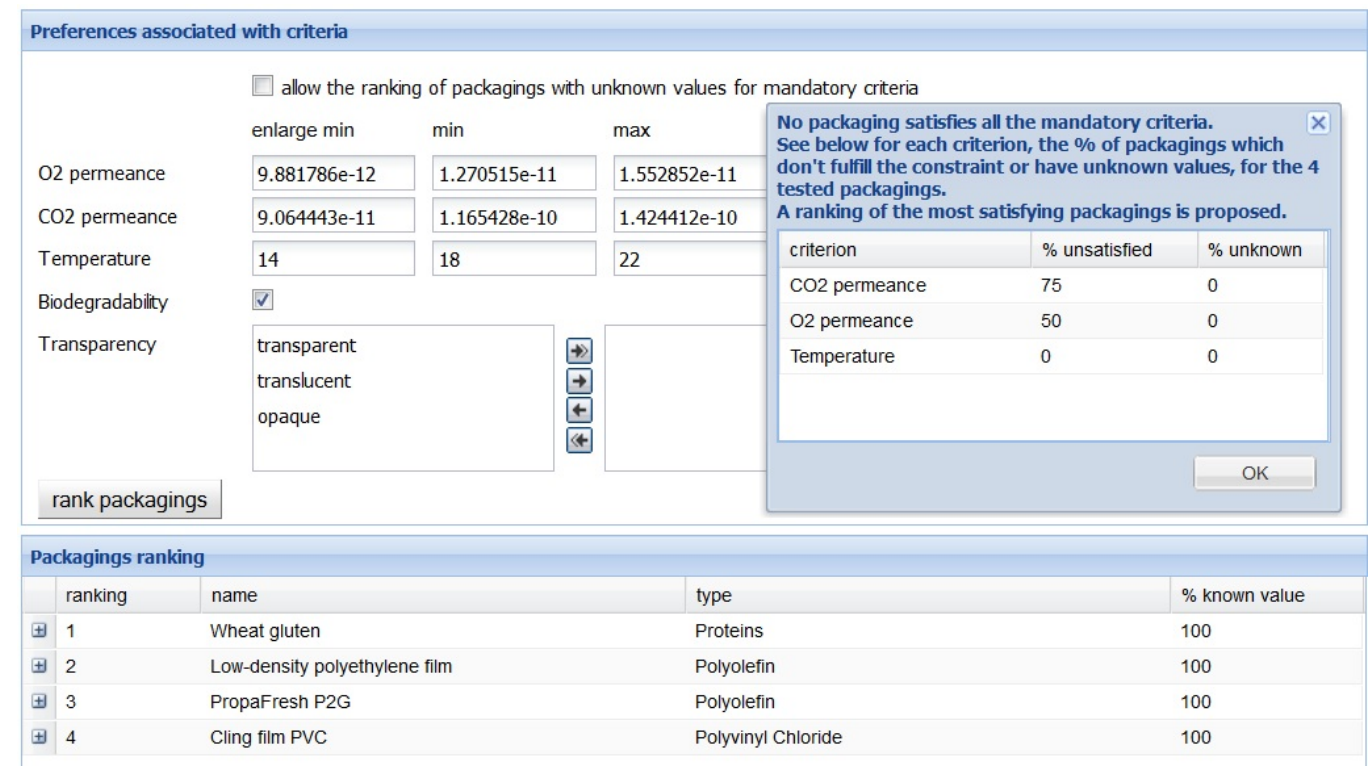

Figure 9: List of the closest packagings to the ideal one in the case of Apricot. 


\section{Conclusion and prospect}

In this paper, we proposed a Decision Support System (DSS) for selecting packaging material for fresh fruits and vegetables which relies on an interdisciplinary approach, coupling process engineering with knowledge engineering and computer science. Mass transfer models aiming at realizing virtual MAP simulations are gathered with packaging and fresh produce databases in a web-application. A bipolar querying module was developed in order to allow the user querying the packaging database starting from food requirements such as mass transfer properties and other criteria like transparency, biodegradability, cost, etc. If several packaging materials are in the same equivalent class after having satisfied the constraints, a refined ranking is proposed on the wishes. In the querying process, the imprecision associated with the data stored in the database is taken into account thanks to the use of comparison degrees between user preferences and data. If none packaging material satisfies the query, the DSS gives a ranking of the less distant packaging material from the target constraints.

Compared to the current stakeholder decision-making practices, this DSS is a significant breakthrough in the field of food packaging, especially that of fresh respiring produce. As aforementioned, due to their intense respiring metabolism, fresh produce necessitate, for their packaging material, specific values of $\mathrm{O} 2 / \mathrm{CO} 2$ permeabilites, belonging to a narrow and specific range of values, that depends on the nature of the product. In practice, the choice 
of this packaging material is based on the specifications defined by the food manufacturer. To define the specifications of $\mathrm{O} 2 / \mathrm{CO} 2$ permeabilities, experimental trials on various materials with a large panel of gas permeabilites are carried out until the packaging suitable to preserve the food is found. This "pack-and-pray" approach is fully empirical and remains qualitative without any formalization of the link between packaging properties and needs of the food. We can easily imagine the added value that the use of a numerical tool like this DSS would bring to food manufacturers by helping them to choose the right packaging material. In addition to the main criterion associated with food shelf life, the DSS proposed in this paper answers to multi-criteria queries including other food packaging characteristics. Therefore, in addition to the permeabilities criteria, it permits to analyze several other criteria that could be constraints or wishes related to the food manufacturer constraints, acceptances and needs: biodegradability, transparency etc. This type of tool was never attempted previously in that field. Among the list of possible packaging retrieved by the DSS, the user has to choose one (usually the one ranked in first) and then to test it in real condition of use. Compared to the empirical approach that requires numerous experimental trials, using the DSS the user will have only one trial to perform (validation step). For the reasons aforementioned, the DSS proposed in this paper definitively assists decision-making in the field of food packaging for fresh produce.

This work has highlighted the importance of storage databases and of their content. Data associated with new packaging materials developed dur- 
ing the EcoBioCap have been stored in the DSS database. In the framework of the project, the DSS will be used to benchmark the new packagings developed for different case studies (respiring cheese, strawberries and mushrooms) (to be further presented). More generally, it is crucial to fill in databases and especially the packaging database with a maximum of data in order to have a panel of materials representative of the different possibilities available on the market and in $\mathrm{R} \& \mathrm{D}$. As the manual entering of data in databases is a time consuming task, methodologies coming from the computer science field allowing the semi-automatic "feeding" of databases from documents founds on the Web should be developed and is a perspective of this work. 
498

499

500

\section{References}

[Bordogna and Pasi, 1994] Bordogna, G. and Pasi, G. (1994). A fuzzy query language with a linguistic hierarchical aggregator. In Proceedings of the 1994 ACM symposium on Applied computing, SAC '94, pages 184-187, New York, NY, USA. ACM.

[Buche et al., 2011] Buche, P., Couvert, O., , Dibie-Barthelemy, J., Hignette, G., Mettler, E., and Soler, L. (2011). Flexible querying of web data to simulate bacterial growth in food. Food Microbiology, 28(4):685-693.

[Cagnon et al., 2012] Cagnon, T., Guillaume, C., Guillard, V., and Gontard, N. (2012). Nano and micro-structuring of materials from a single agropolymer for sustainable MAP preservation of fresh food. Packaging Technology and Science, (26):137-148.

[Cagnon et al., 2013] Cagnon, T., Guillaume, C., Guillard, V., and Gontard, N. (2013). Fresh food packaging design: A requirement driven approach applied to strawberries and agro-based materials. Innovative Food Science E6 Emerging Technologies, (20):288-298.

[Charles et al., 2003] Charles, F., Sanchez, J., and Gontard, N. (2003). Active modified atmosphere packaging of fresh fruits and vegetables: Modeling with tomatoes and oxygen absorber. Journal of Food Science, 68(5):1736-1742. 
[Charles et al., 2005] Charles, F., Sanchez, J., and Gontard, N. (2005). Modeling of active modified atmosphere packaging of endives exposed to several postharvest temperatures. Journal of Food Science, 70(8):E443-E449.

[de Tré et al., 2009] de Tré, G., Zadrozny, S., Matthé, T., Kacprzyk, J., and Bronselaer, A. (2009). Dealing with positive and negative query criteria in fuzzy database quering bipolar satisfaction degrees. LNAI, FQAS, 5822:593-604.

[Destercke et al., 2011] Destercke, S., Buche, P., and Guillard, V. (2011). A flexible bipolar querying approach with imprecise data and guaranteed results. Fuzzy sets and Systems, 169:51-64.

[Dubois and Prade, 1988] Dubois, D. and Prade, H. (1988). Possibility theory- An approach to computerized processing of uncertainty. Plenum Press, New York, USA.

[Dubois and Prade, 1997] Dubois, D. and Prade, H. (1997). The three semantics of fuzzy sets . Fuzzy Sets and Systems, 90(2):141-150.

[Dubois and Prade, 2002a] Dubois, D. and Prade, H. (2002a). Bipolarité dans un processus d'interrogation flexible. In Rencontres francophones sur la Logique Floue et ses Applications, LFA.

[Dubois and Prade, 2002b] Dubois, D. and Prade, H. (2002b). Bipolarity in flexible querying. LNAI, 2522:174-182. 
[Dubois and Prade, 2008] Dubois, D. and Prade, H. (2008). An introduction to bipolar representations of information and preference. International Journal of Intelligent Systems, 23:866-877.

[Floros and Matsos, 2005] Floros, J. D. and Matsos, K. I. (2005). Introduction to modified atmosphere packaging. In Han, J., editor, Innovations in food packaging, pages 159-172. Elsevier academic press, New York.

[Guilbert et al., 2011] Guilbert, S., Guillaume, C., and Gontard, N. (2011). New Packaging Materials Based on Renewable Resources: Properties, Applications, and Prospects. Ajjguilera, J M and BarbosaCanovas, G V and Simpson, R and WeltiChanes, J and BermudezAguirre, D.

[Guillaume et al., 2008] Guillaume, C., Chalier, P., and Gontard, N. (2008). Modified atmosphere packaging using environmentally compatible and active food packaging materials. In Chiellini, E., editor, Environmentally compatible food packaging, chapter 16, pages 396-418. CRC Press.

[Guillaume et al., 2010] Guillaume, C., Schwab, I., Gastaldi, E., and Gontard, N. (2010). Biobased packaging for improving preservation of fresh common mushrooms (Agaricus bisporus L.). Innovative Food Science $\&$ Emerging Technologies, 11(4):690-696.

[Haemmerlé et al., 2007] Haemmerlé, O., Buche, P., and Thomopoulos, R. (2007). The MIEL system: Uniform interrogation of structured and weakly-structured imprecise data. J. Intell. Inf. Syst., 29(3):279-304. 
[Liétard et al., 2011] Liétard, L., Tamani, N., and Rocacher, D. (2011). Fuzzy bipolar conditions of type "or else". In FUZZ-IEEE, pages 25462551.

[Mahajan et al., 2007] Mahajan, P. V., Oliveira, F. A. R., Montanez, J. C., and Frias, J. (2007). Development of user-friendly software for design of modified atmosphere packaging for fresh and fresh-cut produce. Innovative Food Science 83 Emerging Technologies, 8(1):84-92.

[Souza-Gallagher and Mahajan, 2013] Souza-Gallagher, M. and Mahajan, P. (2013). Integrative mathematical modelling for MAP design of freshproduce: Theoretical analysis and experimental validation. Food Control, $29(2): 444-450$.

[Tamani et al., 2013] Tamani, N., Liétard, L., and Rocacher, D. (2013). A fuzzy ontology for database querying with bipolar preferences. International Journal of Intelligent Systems. Wiley Periodicals, Inc., 28(1):4-36.

[Zadeh, 1965] Zadeh, L. (1965). Fuzzy sets. Information and control, 8(3):338-353.

[Zadrozny and Kacprzyk, 2007] Zadrozny, S. and Kacprzyk, J. (2007). Bipolar queries using various interpretations of logical connectives. LNAI, IFSA, 4529:182-190. 
578 [Zadrozny and Kacprzyk, 2009] Zadrozny, S. and Kacprzyk, J. (2009). Bipo579 lar queries: An approach and its various interpretations. In In Proceedings 580 of IFSA/EUSFLAT, pages 1288-1293.

581 [Zagory and Kader, 1988] Zagory, D. and Kader, A. A. (1988). Modified 582 atmosphere packaging of fresh produce. Food Technology, 42(9):70-\&. 